\title{
Multi-modal Function Optimization of Immune Clone Based on Uniform Design

\author{
Bo HU
}

\author{
Science and Technology College Jiangxi Normal University, Nanchang, China \\ hb9012005@163.com
}

\begin{abstract}
Keywords: Immune Optimization, Multimodal Function, Population Distribution, Local Search, Uniform Design.
\end{abstract}

\begin{abstract}
In order to obtain all the optimal solutions of multimodal functions as much as possible, a multi peak function optimization based on uniform design is proposed. The algorithm adopts uniform design to initialize the population and ensure the uniformity and diversity of the initial antibody population distribution. Larmark learning strategy is used to search the local population in order to enhance the convergence speed and precision of the algorithm. In the immune clonal parameter setting, the parameter setting problem is described as a multi factor and multi level uniform design problem. Experimental results show that the algorithm is better in finding the best.
\end{abstract}

\section{Introduction}

In real life, a lot of problems after mathematical modeling can be abstracted as a function optimization problem. At the same time, due to the complexity of the problem, many problems are modeled after the multi peak function optimization problem ${ }^{[1,2]}$, that is, the need to find a number of global optimal solutions and local optimal solution, so as to provide a variety of choices for the decision maker. Therefore, how to obtain the global maximum point and the maximum value of the multi peak function as much as possible has been the subject of continuous exploration.

Artificial immune system is by the mechanisms of biological immune inspired the establishment of a used to solve a variety of complex issues of computing systems, multiple fields already in optimization; network security, intelligent control and data processing are widely used ${ }^{[3]}$. Immune clone algorithm is one of the main algorithms of artificial immune system, and it has a good effect on solving the function optimization problem ${ }^{[4]}$. In order to further enhance the searching ability, there are many scholars experimental design method is introduced to the immune clonal algorithm in, such as orthogonal immune design ${ }^{[5,6]}$, in the single objective of high-dimensional multi valued function optimization showed very good global search ability. Some steps of the immune algorithm can be considered from the experimental point of view ${ }^{[6]}$. Based on this, in this paper, the use of uniform design to initialize the population, combined with the characteristics of multi peak function, using based on Lamarckian local search strategy for solving multimodal function optimization problems. Experimental results show that the algorithm can find more local optimal values, and the solution is faster.

\section{Key Technologies}

\section{Uniform Design and Advantages}

Uniform design is an effective method to solve multi - factor and multi - level experimental problems. Uniform design and orthogonal design, belong to the part of the test method, but its more consideration test points are uniformly distributed in the range of experiment, the minimal test to get the most information ${ }^{[7,8]}$, and test times than that of the orthogonal design method was significantly reduced, which makes uniform design is particularly suited for in multivariate multilevel test. 
In general, if there are $N$ factors, and each factor has $Q$ levels, there is a total of $Q^{N}$ species combination. When $N$ and $Q$ are relatively large, it is not possible to take all the combinations of experiments. Therefore, it is needed to select a small number of representative factors to do the sample. The uniform design method is a kind of strategy, which can effectively reduce the number of experiments.

\section{Initial Population Generation Based on Uniform Design}

For high dimensional multimodal function optimization problems, due to the function itself has multiple poles, and the position of the most advantage of global function is unknown. Therefore, in population initialization process should be as far as possible, so that the initial population uniformly covers the whole feasible region. The method of randomly generated antibody cannot guarantee the uniform distribution of the generated antibody in the solution space, and the search efficiency is lower $^{[9]}$. Therefore, according to the characteristics of uniform distribution, this paper uses uniform design for population initialization. The uniformity of initial population distribution ensures the population diversity, so that the algorithm can converge at a faster rate in the global range.

The specific process is as follows:

In this paper, $x=\left(x_{1}, x_{2}, \cdots, x_{n}\right)$, an antibody that directly represents the problem, that is, the problem is not encoding, Antibodies direct use of the original form of the variable

The definition of $U_{n}\left(q^{s}\right)$ repre uniform design, where $\mathrm{n}$ is the number of experiments, $\mathrm{q}$ is the number of levels, and $S$ is the number of columns (the maximum number of factors). Uniform design, the number of trials and the level of the same, so in this paper, the uniform design is recorded as $U_{n}\left(q^{s}\right)$.

In clonal optimization, if the uniform design table as a population, uniform table of each line as a antibody on the characterization of the individual, is uniform test design should take into account factors corresponds to in the antibody gene.

There are many methods to construct uniform design table, such as the good lattice point method, the congruence Latin method and the optimization method and so on ${ }^{[8]}$. In this paper, we use the grid point method to construct uniform design table. Please refer to the literature ${ }^{[8]}$.

Due to the uniform design is suitable for discrete variables, not suitable for continuous variables.

Therefore, it is assumed that each antibody contains $N$ factor, defined by $x_{i}$ as the first $i$ factor, and each factor is discretized into a finite number of values. Specifically, the interval of the

$x_{i}$ into $q_{i}$ level, and take the level of $a_{i, 1}, a_{i, 2}, \cdots, a_{i, q i}$, Using the good grid point method of literature [8] to construct uniform design table $U_{n}\left(q^{s}\right)$, we can get the $q$ antibody in $q^{s}$ combination. This $q$ antibody was used as the initial population.

\section{Larmark Lamarckian Learning}

For multimodal function optimization problems, it should be as much as possible to find the local optimal value. Therefore, it is necessary to design a local search strategy. Here, the use of Lamarck learning to perform local search.

Lamarck evolution theory believes that the use of waste into the back, which is acquired, the individual acquired learning traits can be directly fed back on the gene, through the gene to offspring ${ }^{[10,11]}$. Local search can be regarded as an individual's acquired learning process, the search to a good antibody fragments directly to the antibody, and modify the individual's fitness, through the genetic role to the next generation. Therefore, the local search is the learning process of the parents in their neighborhood, which can guide the individual to move to a better solution.

Specific as follows:

The original affinity of antibody $A_{i}$ was $f_{\left(A_{i}\right)}$, and the affinity of antibody was $f^{\prime}{ }_{\left(A_{i}\right)}$, 
$f^{\prime}{ }_{\left(A_{i}\right)}=\eta f_{\left(A_{i}\right)}$. Among them, $\eta$ is a random number which follows the normal distribution $(1<\eta<5)$. If $f_{\left(A_{i}\right)}^{\prime}>f_{\left(A_{i}\right)}$ and $f_{\left(A_{i}^{\prime}\right)}^{\prime}>f_{\text {best }(t)}$, then learn to succeed, with antibody gene $A_{i}{ }^{\prime}$ to replace ${ }^{A_{i}}$. Among them, $f_{\text {best }(t)}$ was the highest degree of fitness for the $t$ generation.

\section{Algorithm Implementation}

This paper designed the immune cloning algorithm implementation steps are as follows: (Step1-Step5):

Step1 Using uniform design initialization population, set up an evolutionary algebra for $\mathrm{G}$, so that $\mathrm{g}=0$ calculation of all the individual's affinity (affinity function that is to optimize the function itself);

Step2 Takes the evolution algebra $g$ as termination condition to judge whether it satisfies the end condition. If the end condition is satisfied, then the program is terminated, the output of the optimal solution; otherwise Step3;

Step3 According to the affinity of each antibody was cloned and amplified. The clone adopts the adaptive clone [11], namely the high affinity antibody clone number is more;

Step4 Adaptive mutation ${ }^{[12]}$ to clone population; calculate the affinity of antibody, and the high affinity antibody for Lamarck study (see Section 2.3);

Step5 $g=g+1$ turn Step2.

\section{Simulation Experiment and Result Analysis}

\section{Test Function and Analysis}

Under the Windows operating system, using Matlab9.0

The algorithm is programmed to achieve, and compared with the literature ${ }^{[2]}$ analysis.

\section{Parameter Setting of immune Clone Algorithm}

The parameter setting of immune clonal algorithm is usually determined by experience and experiment, so the experiment workload is large and it is difficult to get the best parameter combination $^{[13]}$. In this paper, the parameter setting of the immune algorithm is described as a multi factor and multi level test design in uniform design. The maximum number of factors in uniform design can be immune clonal algorithm parameters; the number of levels is the range of values for each parameter. In this paper, the parameters of the clonal selection algorithm are mainly mutation probability $p_{m}$, cloning proportion coefficient $k$, learning coefficient $\eta$, population size $M$.

The basic clonal selection algorithm of the three parameters $(\mathrm{s}=4)$, if each parameter takes 10 values $(n=10)$. The steps of using uniform design to set algorithm parameters are briefly described as follows:

(1) Determine the range of values for $s=4$ parameters, respectively;

(2) For each parameter to take $n=10$ level, according to the uniform design of the recommended table, get $U_{n}\left(n^{5}\right)$;

(3) According to the test plan of uniform table, find out the corresponding parameter combination, carry on a number of tests to each group, and statistics the data, and find out the best combination.

This paper, set the population size, mutation probability, cloning coefficient, learning coefficient were $[30,80],[0.35,0.85],[0.1,0.4]$. $[2,4],[2,5]$, by the uniform experimental design, final parameters are as follows: population $\mathrm{M}=60, p_{m}=0.3$, coefficient cloning $k=2$, learning coefficient $\eta=1.2,200$ times the maximum number of evolution. Each algorithm is run 100 times, and the results are shown in table 1. 
In order to verify the performance of this algorithm, we use 6 commonly used Benchmark functions to test (for the minimum value):

(1) Schaffer function

$$
f_{1}(x)=\frac{\sin ^{2} \sqrt{x_{1}^{2}+x_{2}^{2}}-0.5}{\left[1+0.001\left(x_{1}^{2}+x_{2}^{2}\right)\right]^{2}}-0.5, x \in[-100,100]
$$

(2) Shubert

$$
f_{2}(x)=\sum_{i=1}^{5} i \cdot \cos \left((i+1) \cdot x_{1}+i\right) \cdot \sum_{j=1}^{5} j \cdot \cos \left((j+1) \cdot x_{2}+j\right), x_{i} \in[-10,10]
$$

(3) Rastrigin function

$$
f_{3}(x)=\sum_{i=1}^{D}\left[x_{i}^{2}-10 \cos \left(2 \pi x_{i}\right)+10\right], x_{i} \in[-5.12,5.12]
$$

(4) Griewank function

$$
f_{4}(x)=\frac{1}{4000} \sum_{i=1}^{D}\left(x_{i}^{2}\right)-\prod_{i=1}^{D} \cos \left(x_{i} / \sqrt{i}\right)+1, x_{i} \in[-600,600]
$$

(5) Schwefel function

$$
f_{5}(x)=418.9829 \times D-\sum_{i=1}^{D} x_{i} \sin \left(\left|x_{i}\right|^{1 / 2}\right), \quad x_{i} \in[-500,500]
$$

(6) Rosenbrock function

$$
f_{6}(x)=\sum_{i=1}^{D}\left[100\left(x_{i+1}-x_{i}^{2}\right)^{2}+\left(x_{i}-1\right)^{2}\right], x_{i} \in[-3,3]
$$

The test functions, Schaffer function is with strong oscillation of multi function, optimality theory value is -1 ; Shubert function within the search area about 760 local extreme points and 18 global optimum, the optimal theoretical value for -186.7309 ; rastrigrin function for multi extreme value function, in the solution space exists about $10 \mathrm{~N}$ ( $\mathrm{n}$ is the solution space dimension) local minima, the theoretical optimal value of 0 ; Griewank function has many local extremum, in $(0, \ldots$ (0) obtains the global minimum 0; the Schwefel function is the multi extreme value function, in (420.96). Obtain the theoretical optimal value of 420.96) $0[14,15]$.

Tab.1 Performance Comparison of Correlation Algorithm

\begin{tabular}{ccccccc}
\hline function & dimension & algorithm & mean value & $\begin{array}{c}\text { standard } \\
\text { deviation }\end{array}$ & $\begin{array}{c}\text { Convergence } \\
\text { rate }\end{array}$ & Time/s \\
\hline \multirow{2}{*}{ Schaffer } & 2 & Literature [2] & -0.9493 & $1.8961 \mathrm{e}-01$ & $34 / 100$ & 17.2 \\
& & in this paper & -0.9618 & 0 & $100 / 100$ & 12.2 \\
\hline \multirow{2}{*}{ Shubert } & 2 & Literature [2] & -172.0092 & $4.5088 \mathrm{e}+01$ & $0 / 100$ & 23.5 \\
& \multirow{2}{*}{$\begin{array}{c}* \\
\text { Rastrigin }\end{array}$} & in this paper & -184.7121 & 0 & $100 / 100$ & 18.5 \\
\hline \multirow{2}{*}{ Griewank } & 10 & Literature [2] & 48.5893 & $1.9544 \mathrm{E}-03$ & $82 / 100$ & 19.8 \\
& & in this paper & 37.9846 & $2.4163 \mathrm{E}-07$ & $100 / 100$ & 15.3 \\
\hline \multirow{2}{*}{ Schwefel } & \multirow{2}{*}{10} & Literature [2] & 40.2196 & $1.2911 \mathrm{E}-01$ & $78 / 100$ & 21.0 \\
& & in this paper & 10.1537 & 0 & $98 / 100$ & 18.1 \\
\hline
\end{tabular}

The experimental results show that the algorithm has high precision, and the probability of finding the optimal solution in a finite algebra is larger than that of the optimal solution. In addition, the variance of the algorithm is small, which shows that the algorithm is stable. The time of the algorithm to find the optimal solution is shorter, which shows that the various solution operators designed in this paper are effective. 


\section{Conclusions}

In this paper, we propose an immune clonal algorithm based on uniform design to solve multimodal function optimization. Using uniform design for population initialization and setting algorithm parameters, the local search mechanism based on LARMARK is used to enhance the local search capability. The simulation results show that the algorithm is effective.

\section{References}

[1] Ye Wen, Ouyang Zhonghui, Zhu Aihong, Fan Honda. Solving the optimization function of niche clonal selection algorithm [J]. Systems engineering and electronics, 2010, 23 (4): 124-126. (5): $210-214$.

[2] Lu Qing, Liang Changyong, Yang Shanlin, Zhang Junling. Adaptive niche genetic algorithm for multi valued function optimization [J] pattern recognition and artificial intelligence, 2009, 43 (2): 86-91.

[3] M.G. Gong, L.C.Jiao, L.N.Zhang, H.F. Du Immune secondary response and clonal selection inspired optimizers [J]. Progress in Natural Science 2009, 19: 237-253.

[4] Yu Hang, Jiao Licheng, Gong Maoguo, Yang Dongdong. Optimization of clonal selection function based on orthogonal experimental design. Journal of software [J], 2010, 21 (5): 950- 967.

[5] Jiang Zhongyang, Cai Zixing, Wang Yong. A hybrid adaptive orthogonal genetic algorithm for solving global optimization problems [J]. Journal of software, 2010, 21 (6): 1296-1307.

[6] Cai Zixing, central Jiang, Wang Yong, Luo Yidan. A new evolutionary algorithm [J]. Journal of computer optimization based on orthogonal experimental design constraints, 2010, 33 (5): 855 - in.

[7] Fan Tiehu, Qin Guihe and Zhao Qi. A distributed search algorithm for solving the uniform design and transformation of BLX- for continuous optimization problems [J]. Computer research and development, 2011, 48 (6):1049 - 1058.

[8] Fang Kaitai, Ma Changxin. Orthogonal and uniform design [M]. Beijing: Science Press, 2009.

[9] Liang Changyong, Lu Qing, Zhang Enqiao, Wei Zhaowang. Research on multi agent genetic algorithm based on uniform design [J]. Journal of systems engineering 2009, 32 (2): 11-18.

[10] Gong Maoguo, Jiao Lichen,Liu Fang, Yang Jie. Memetic computation based on regulation between neural and immune systems: Framework and a case study.Science China the (Sciences Information), 2010, 45 (11): 2131-2138.3082-3093.

[11] Xia Zhuchang, Liu Fang, Gong Maoguo, Qi Yutao. Based on memory Lamarckian evolutionary algorithm for job shop scheduling [J], 2010, 21 (1): 1-5. (12):

[12] Wang Yong, Cai Zi-Xing, Zhou Yu-Ren, Zeng Wei. An adaptive trade-off model for constrained evolutionary Zeng optimization [J]. IEEE Transactions on Evolutionary Computation, 2008, 12 (1): 80-92

[13] Wang Rongfang, Jiao Licheng, Yang Shuyuan, Liu Fang. The natural computation method for adaptive dynamic control population size [J]. Journal of software, 2012, 23 (7): 1760-1772.

[14] Zhang Ke. Parameter adjustment of ant colony algorithm based on uniform design and chaos theory [J], computer engineering, 2012, 38 (14): 144 - 141

[15] Mao-Guo Gong, Li-Cheng Jiao. Du Immune secondary response and clonal selection inspired optimizers [J]. Progress in Natural Science 2009, 19: 237-253. 